\title{
Fixed Point Theorems of Set-Valued Mappings in Partially Ordered Hausdorff Topological Spaces
}

\author{
Shujun Jiang, ${ }^{1}$ Zhilong $\mathrm{Li}^{2}$ and Shihua $\mathrm{Luo}^{2}$ \\ ${ }^{1}$ Department of Mathematics, Jiangxi University of Finance and Economics, Nanchang 330013, China \\ ${ }^{2}$ School of Statistics, Jiangxi University of Finance and Economics, Nanchang 330013, China \\ Correspondence should be addressed to Zhilong Li; lzl771218@sina.com
}

Received 19 December 2013; Accepted 20 February 2014; Published 30 March 2014

Academic Editor: Salvador Romaguera

Copyright (c) 2014 Shujun Jiang et al. This is an open access article distributed under the Creative Commons Attribution License, which permits unrestricted use, distribution, and reproduction in any medium, provided the original work is properly cited.

In this work, several fixed point theorems of set-valued monotone mappings and set-valued Caristi-type mappings are proved in partially ordered Hausdorff topological spaces, which indeed extend and improve many recent results in the setting of metric spaces.

\section{Introductions}

In 1976, Caristi [1] proved Caristi's fixed point theorem [1, 2], which has been the subject of intensive research in the past decades, and has found many applications in nonlinear analysis. Recall that this general fixed point theorem states that each mapping $T: X \rightarrow X$ has a fixed point provided that $(X, d)$ is a complete metric space and there exists a lower semicontinuous and bounded below function $\varphi: X \rightarrow$ $\mathbb{R}$ such that $d(x, T x) \leq \varphi(x)-\varphi(T x)$ for each $x \in X$. Kirk [2] gave an elegant proof of primitive Caristi's result by investigating the existence of maximal elements of a partially ordered metric space $(X, \preceq)$, where $\preceq$ is a partial order defined by

$$
x \leq y \Longleftrightarrow d(x, y) \leq \varphi(y)-\varphi(x), \quad \forall x, y \in X .
$$

Since then, Kirk's method has been widely used in the generalizations of primitive Caristi's result and the study of fixed point theorems of monotone mappings with respect to a partial order introduced by a functional and many satisfactory fixed point results have been obtained in metric spaces (see [3-10]).

The purpose of this paper is to generalize the results of [3-10] to general topological spaces. Under suitable assumptions, we proved several fixed point theorems of set-valued monotone mappings and set-valued Caristi-type mappings in partially ordered Hausdorff topological spaces, which indeed extend and improve many recent results in the setting of metric spaces.

\section{Main Results}

Let $X$ be a Hausdorff topological space and let $\leq$ be a partial order on $X$. For each $x \in X$, let $[x,+\infty)=\{z \in X: x \preceq z\}$ and let $(-\infty, x]=\{z \in X: z \preceq x\}$.

Let $D$ be a nonempty subset of $X$ and $T: X \rightarrow 2^{X}$, where $2^{X}$ denote the family of all nonempty subset of $X$. T is increasing on $D$, if for each $x, y \in D$ with $x \preceq y$ and each $u \in T x$, there exists $v \in T y$ such that $u \preceq v$; $T$ is quasiincreasing on $D$, if for each $x, y \in D$ with $x \preceq y$ and each $v \in T y$, there exists $u \in T x$ such that $u \preceq v$. T has compact value on $D$, if $T x$ is compact for each $x \in D$. T is a Caristitype mapping, if for each $x \in X$, there exists $y \in T x$ such that $x \preceq y$.

Let $\preceq_{1}$ be the inverse partial order of $\preceq$. It is clear that $T$ : $X \rightarrow 2^{X}$ is increasing on $D$ with respect to $\preceq_{1}$ if $T$ is quasiincreasing on $D$ with respect to $\preceq$.

In this paper, we make the following assumptions:

$\left(A_{1}\right)$ for each totally ordered set $N \subset X$, there exists a sequence $\left\{x_{n}\right\} \subset N$ such that for each $x \in N$ with $x \neq \sup N$, there exists some $x_{n_{0}} \in\left\{x_{n}\right\}$ such that $x \leq x_{n_{0}} ;$

$\left(A_{2}\right)$ for each totally ordered set $N \subset X$, there exists a sequence $\left\{x_{n}\right\} \subset N$ such that for each $x \in X$ with 
$x \neq \inf N$, there exists some $x_{n_{0}} \in\left\{x_{n}\right\}$ such that $x_{n_{0}} \leq x$;

$\left(A_{3}\right)$ for each increasing sequence $\left\{x_{n}\right\} \subset X$, there exists a subsequence $\left\{x_{n_{k}}\right\} \subset\left\{x_{n}\right\}$ and some $x \in X$ such that $x_{n_{k}} \rightarrow x$

$\left(A_{4}\right)$ for each decreasing sequence $\left\{x_{n}\right\} \subset X$, there exists a subsequence $\left\{x_{n_{k}}\right\} \subset\left\{x_{n}\right\}$ and some $x \in X$ such that $x_{n_{k}} \rightarrow x$

$\left(A_{5}\right)$ for each $\left\{x_{n}\right\},\left\{y_{n}\right\} \subset X$ with $x_{n} \leq y_{n}$ for each $n$, if there exists some $x, y \in X$ such that $x_{n} \rightarrow x$ and $y_{n} \rightarrow y$, then $x \leq y$;

$\left(A_{6}\right)$ for each sequence $\left\{x_{n}\right\} \subset X$ with $y \preceq x_{n}$ for some $y \in X$ and each $n$, if there exists $x \in X$ such that $x_{n} \rightarrow x$, then $y \leq x$;

$\left(A_{7}\right)$ there exists a functional $\varphi: X \rightarrow \mathbb{R}$ such that

$$
\varphi(y)<\varphi(x), \quad \forall x, y \in X, x \preceq y, x \neq y .
$$

Theorem 1. Let $X$ be a Hausdorff topological space, let $\leq$ be a partial order on $X$, and let $T: X \rightarrow 2^{X}$. Assume that $\left(A_{1}\right)$, $\left(A_{3}\right)$, and $\left(A_{5}\right)$ are satisfied. If there exists $x_{0} \in X$ such that $T x_{0} \cap\left[x_{0},+\infty\right) \neq \varnothing, T$ is increasing and has compact value on $\left[x_{0},+\infty\right)$. Then $T$ has a maximal fixed point in $x^{*} \in\left[x_{0},+\infty\right)$; that is, let $x$ be a fixed point of $T$ in $\left[x_{0},+\infty\right)$ such that $x^{*} \leq x$, and then $x=x^{*}$.

Proof. Let

$$
Q=\left\{x \in\left[x_{0},+\infty\right): T x \cap[x,+\infty) \neq \varnothing\right\} .
$$

Clearly, $Q$ is nonempty since $x_{0} \in Q$. We divide the proof into four steps.

Step 1. We show that $\left(A_{3}\right)$ holds on $Q$. Let $\left\{x_{n}\right\} \subset Q$ be an arbitrary increasing sequence. By $\left(A_{3}\right)$, there exists a subsequence $\left\{x_{n_{k}}\right\} \subset\left\{x_{n}\right\}$ and some $x \in X$ such that

$$
x_{n_{k}} \longrightarrow x \text {. }
$$

Let $n_{k} \rightarrow \infty$ in (4), and then by $\left(A_{5}\right)$ we have $x_{0} \leq x$; that is, $x \in\left[x_{0},+\infty\right)$ since $x_{0} \leq x_{n_{k}}$ for each $n_{k}$. For arbitrary given $n_{k_{0}}$, we have $x_{n_{k_{0}}} \leq x_{n_{k}}$ and hence $x_{n_{k_{0}}} \preceq x$ by $\left(A_{5}\right)$. Moreover the arbitrary property of $n_{k_{0}}$ forces the following:

$$
x_{n_{k}} \preceq x, \quad \forall n_{k} .
$$

Since $x_{n_{k}} \in Q$, there exists $y_{n_{k}} \in T x_{n_{k}}$ such that

$$
x_{n_{k}} \preceq y_{n_{k}}, \quad \forall n_{k} .
$$

By (5), $x \in\left[x_{0},+\infty\right)$ and the increasing property of $T$ on $\left[x_{0},+\infty\right)$, there exists $z_{n_{k}} \in T x$ such that $y_{n_{k}} \leq z_{n_{k}}$ for each $n_{k}$. This together with (6) implies that

$$
x_{n_{k}} \preceq z_{n_{k}}, \quad \forall n_{k} .
$$

Since $T$ has compact value on $\left[x_{0}, \infty\right)$, then $T x$ is compact and so there exists a subsequence $\left\{z_{n_{k_{i}}}\right\} \subset\left\{z_{n_{k}}\right\}$ and $z \in T x$ such that

$$
z_{n_{k_{i}}} \longrightarrow z
$$

Note that $x_{n_{k_{j}}} \leq z_{n_{k_{j}}}$ for each $n_{k_{i}}$, and then by (4), (8), and $\left(A_{5}\right)$, we have $x \leq z$. This implies that $z \in T x \cap[x,+\infty) \neq \varnothing$ and so $x \in Q$ since $x \in\left[x_{0},+\infty\right)$. Hence $\left(A_{3}\right)$ holds on $Q$.

Step 2. We show that each increasing sequence $\left\{u_{n}\right\} \subset Q$ has an upper bound in $Q$. Since $\left(A_{3}\right)$ holds on $Q$ by Step 1, there exists a subsequence $\left\{u_{n_{k}}\right\} \subset\left\{u_{n}\right\}$ and some $u \in Q$ such that

$$
u_{n_{k}} \longrightarrow u
$$

Note that for arbitrary given $n, u_{n} \leq u_{n_{k}}$ for all $n_{k} \geq n$, and then by $(9)$ and $\left(A_{5}\right)$ we have $u_{n} \leq u$. Moreover the arbitrary property of $n$ forces $u_{n} \leq u$ for each $n$; that is, $u$ is an upper bound of $\left\{x_{n}\right\}$.

Step 3. We show that each totally ordered set $\left\{x_{\alpha}\right\}_{\alpha \in \Gamma} \subset Q$ has an upper bound in $Q$, where $\Gamma$ is a directed set.

If there exists $x \in\left\{x_{\alpha}\right\}$ such that $x=\sup _{\alpha \in \Gamma} x_{\alpha}$, then $x$ is an upper bound of $\left\{x_{\alpha}\right\}_{\alpha \in \Gamma}$ and hence the proof is finished. Thus we may assume that $x \neq \sup _{\alpha \in \Gamma} x_{\alpha}$ for each $x \in\left\{x_{\alpha}\right\}$. By $\left(A_{1}\right)$, there is a sequence $\left\{x_{\alpha_{n}}\right\} \subset\left\{x_{\alpha}\right\}$ such that there exists $x_{\alpha_{n_{0}}} \in\left\{x_{\alpha_{n}}\right\}$ such that

$$
x \leq x_{\alpha_{n_{0}}} .
$$

Set

$$
u_{n}=\max \left\{x_{\alpha_{1}}, x_{\alpha_{2}}, \ldots, x_{\alpha_{n}}\right\}, \quad \forall n \geq 1 .
$$

Note that $\left\{x_{\alpha}\right\}$ is totally ordered, and then $\left\{u_{n}\right\}$ is well defined and is an increasing sequence such that $x_{\alpha_{n}} \preceq u_{n}$ for all $n$. By step $2,\left\{u_{n}\right\}$ has an upper bound in $Q$; denote it by $\bar{x}$. Moreover by (10) and (11),

$$
x \leq x_{\alpha_{n_{0}}} \leq u_{n_{0}} \preceq \bar{x}, \quad \forall x \in\left\{x_{\alpha}\right\},
$$

which implies that $\bar{x}$ is an upper bound of $\left\{x_{\alpha}\right\}$.

Step 4. We show that $T$ has a maximal fixed point in $\left[x_{0},+\infty\right)$. By Zorn's lemma, $(Q, \leq)$ has a maximal element $x^{*}$; that is, for each $x \in Q$ with $x^{*} \preceq x$, we must have $x=x^{*}$. Since $x^{*} \in Q$, there exists $y^{*} \in T x^{*}$ such that $x^{*} \leq y^{*}$. Moreover by the increasing property of $T$ on $\left[x_{0},+\infty\right)$, there exists $z^{*} \in T y^{*}$ such that $y^{*} \preceq z^{*}$. This indicates that $z^{*} \in T y^{*} \cap\left[y^{*},+\infty\right) \neq \varnothing$ and hence $y^{*} \in Q$. Finally the maximality of $x^{*}$ in $Q$ implies that $x^{*}=y^{*} \in T x^{*}$; that is, $x^{*}$ is a maximal fixed point of $T$ in $\left[x_{0},+\infty\right)$. The proof is complete.

Theorem 2. Let $X$ be a Hausdorff topological space, let $\leq$ be a partial order on $X$, and let $T: X \rightarrow 2^{X}$. Assume that $\left(A_{2}\right),\left(A_{4}\right)$, and $\left(A_{5}\right)$ are satisfied. If there exists $x_{0} \in X$ such that $T x_{0} \cap\left(-\infty, x_{0}\right] \neq \varnothing, T$ is quasi-increasing and has compact value on $\left(-\infty, x_{0}\right]$. Then $T$ has a minimal fixed point in $x_{*} \in\left(-\infty, x_{0}\right]$; that is, let $x$ be a fixed point of $T$ in $\left(-\infty, x_{0}\right]$ such that $x \leq x_{*}$, and then $x=x_{*}$.

Proof. Let $\preceq_{1}$ be the inverse partial order of $\preceq$. It is clear that $\left(A_{1}\right)$ and $\left(A_{3}\right)$ are satisfied with respect to $\unlhd_{1}$ by $\left(A_{2}\right)$ and 
$\left(A_{4}\right)$. Set $[x,+\infty)_{\unlhd_{1}}=\left\{z \in X: x \preceq_{1} z\right\}$ and $(-\infty, x]_{\unlhd_{1}}=$ $\left\{z \in X: z \preceq_{1} x\right\}$. Obviously, $[x,+\infty)_{\unlhd_{1}}=(-\infty, x]$, $(-\infty, x]_{\varliminf_{1}}=[x,+\infty), T x_{0} \cap\left[x_{0},+\infty\right)_{\unlhd_{1}} \neq \varnothing$, and $T$ is increasing and has compact value on $\left[x_{0},+\infty\right)_{\unlhd_{1}}$. Applying Theorem 1 on $\left(X, \varsigma_{1}\right)$, we find that $T$ has a maximal fixed point $x^{*} \in\left[x_{0},+\infty\right)_{\unlhd_{1}}=\left(-\infty, x_{0}\right]$ corresponding to $\preceq_{1}$. Let $x \in\left(-\infty, x_{0}\right]$ be a fixed point of $T$. If $x \preceq x^{*}$, then $x^{*} \preceq_{1} x$ and hence $x=x^{*}$ by the maximality of $x^{*}$ corresponding to $\preceq_{1}$; that is, $x^{*}$ is a minimal fixed point of $T$ in $\left(-\infty, x_{0}\right]$ corresponding to $\preceq$. The proof is complete.

Example 3. Let $X=\{0\} \cup\{1 / n: n=1,2,3, \ldots\}$ with the usual metric $d(x, y)=\|x-y\|$ for each $x, y \in X$ and the usual order $x \preceq y \Leftrightarrow x \leq y$ for each $x, y \in X$. It is easy to check that $\left(A_{1}\right)-\left(A_{5}\right)$ are satisfied.

Let $T: X \rightarrow 2^{X}$ be defined by

$$
\begin{array}{ll}
T 0=0, & T 1=X, \\
T \frac{1}{n}=\frac{1}{n+1}, & n \geq 2 .
\end{array}
$$

Clearly, $T$ is an increasing mapping and has compact value on $X$. Note that $0 \preceq T 0$ and $[0,+\infty)=X$; then by Theorem $1, T$ has a maximal fixed point $x=1$.

Let $K: X \rightarrow 2^{X}$ be defined by

$$
\begin{aligned}
& K 0=X, \quad K 1=1, \\
& K \frac{1}{n}=\frac{1}{n+1}, \quad n \geq 2 .
\end{aligned}
$$

Clearly, $K$ is a quasi-increasing mapping and has compact value on $X$. Note that $K 1 \preceq 1$ and $(-\infty, 1]=X$; then by Theorem $2, K$ has a minimal fixed point $x=0$.

The following theorem extends primitive Caristi's result to Hausdorff topological spaces.

Theorem 4. Let $X$ be a Hausdorff topological space and let $\preceq$ be a partial order on $X$. Assume that $\left(A_{1}\right),\left(A_{3}\right)$, and $\left(A_{6}\right)$ are satisfied. Then each set-valued Caristi-type mapping $T: X \rightarrow$ $2^{X}$ has fixed point in $X$.

Proof. In analogy to Step 2 in the proof of Theorem 1, by $\left(A_{3}\right)$ and $\left(A_{6}\right)$, we can prove that each increasing sequence $\left\{u_{n}\right\} \subset X$ has an upper bound in $X$. Thus following Step 3 in the proof of Theorem 1, each totally ordered chain of $X$ has an upper bound by $\left(A_{1}\right)$. Moreover by Zorn's lemma, $(X, \preceq)$ has a maximal element; denote it by $x^{*}$. Note that there exists some $y^{*} \in T x^{*}$ such that $x^{*} \preceq y^{*}$; then $x^{*}=y^{*}$ by the maximality of $x^{*}$ and hence $x^{*}$ is a fixed point of $T$. The proof is complete.

The following lemma shows that the conditions $\left(A_{1}\right)$ and $\left(A_{2}\right)$ are not hard to be satisfied.

Lemma 5. Let $X$ be a nonempty set and let $\preceq$ be a partial order on X. If $\left(A_{7}\right)$ is satisfied and $\varphi$ is bounded below (resp., bounded above), then $\left(A_{1}\right)$ (resp., $\left.\left(A_{2}\right)\right)$ is satisfied.

Proof. We only show $\left(A_{1}\right)$ is satisfied, and the proof of the other case is similar.
Let $\left\{x_{\alpha}\right\}_{\alpha \in \Gamma}$ be a totally ordered set of $X$, where $\Gamma$ is a directed set, and set $M=\left\{\varphi\left(x_{\alpha}\right): \alpha \in \Gamma\right\}$. Note that $\varphi$ is bounded below; then inf $M$ exists, and so there exists a subset $\left\{\alpha_{n}\right\}$ of $\Gamma$ such that

$$
\lim _{n \rightarrow \infty} \varphi\left(x_{\alpha_{n}}\right)=\inf M
$$

Let $\beta \in \Gamma$ be an element such that $x_{\beta} \neq \sup _{\alpha \in \Gamma} x_{\alpha}$; then $\varphi\left(x_{\beta}\right) \neq \inf M$ by $\left(A_{7}\right)$. Suppose that $x_{\alpha_{n}} \preceq x_{\beta}$ for each $n$. By $\left(A_{7}\right), \varphi\left(x_{\beta}\right) \leq \varphi\left(x_{\alpha_{n}}\right)$ for each $n$, and consequently, we have $\varphi\left(x_{\beta}\right)=\inf M$ by (15). This is a contradiction, and so there exists some $n_{0}$ such that

$$
x_{\beta} \preceq x_{\alpha_{n_{0}}} .
$$

This shows that $\left(A_{1}\right)$ is satisfied. The proof is complete.

Remark 6. It follows from Lemma 5 that Theorems 1-4 are still valid while $\left(A_{1}\right)$ (resp., $\left(A_{2}\right)$ ) is replaced with $\left(A_{7}\right)$ provided that $\varphi$ is bounded below (resp., bounded above).

\section{Applications to Metric Spaces}

In this section, we shall show that most of the fixed point results in the setting of metric spaces of [3-10] could be derived from Theorems 1-4.

Let $(X, d)$ be a metric space and let $\preceq$ be a partial order on $X$. We list the conditions used in $[3,6,9,10]$ as follows:

$\left(A_{8}\right) \eta: \mathbb{R}_{+} \rightarrow \mathbb{R}_{+}$is a continuous, nondecreasing, and subadditive (i.e., $\eta(t+s) \leq \eta(t)+\eta(s)$ for each $t, s \in$ $\left.\mathbb{R}_{+}\right)$function with $\eta^{-1}(\{0\})=\{0\} ;$

$\left(A_{9}\right)$ there exists a functional $\varphi: X \rightarrow \mathbb{R}$ and a function $\eta: \mathbb{R}_{+} \rightarrow \mathbb{R}_{+}$with $\eta^{-1}(\{0\})=\{0\}$ such that $\eta(d(x, y)) \leq \varphi(x)-\varphi(y)$ for each $x, y \in X$ with $x \preceq y$, where $\preceq$ is a partialorder on $X$;

$\left(A_{10}\right)$ for each $x \in X$, the order interval $\{y \in X: x \preceq y\}$ is closed;

$\left(A_{11}\right)$ for each $x \in X$, the order interval $\{y \in X: y \preceq x\}$ is closed;

$\left(A_{12}\right) \eta: \mathbb{R}_{+} \rightarrow \mathbb{R}_{+}$is nondecreasing.

Remark 7. (i) It is easy to check that $\left(A_{9}\right) \Rightarrow\left(A_{7}\right),\left(A_{10}\right) \Rightarrow$ $\left(A_{6}\right)$, and $\left(A_{10}\right)+\left(A_{11}\right) \Rightarrow\left(A_{5}\right)$.

(ii) Let $\preceq$ be a relation on $X$ defined by

$$
x \preceq y \Longleftrightarrow \eta(d(x, y)) \leq \varphi(x)-\varphi(y), \quad \forall x, y \in X,
$$

where $\eta: \mathbb{R}_{+} \rightarrow \mathbb{R}_{+}$and $\varphi: X \rightarrow \mathbb{R}$. It follows from [3, Lemma 4.1] that $\preceq$ introduced by (17) is a partial order on $X$ provided that $\left(A_{8}\right)$ is satisfied. Clearly, the partial order $\preceq$ on $X$ introduced by (17) is certainly a partial order such that $\left(A_{9}\right)$ is satisfied, and hence $\left(A_{7}\right)$ is satisfied.

Lemma 8. Let $(X, d)$ be a complete metric space and let $\preceq$ be a partial order on $X$. Assume that $\left(A_{9}\right)$ and $\left(A_{12}\right)$ are satisfied. 
Then

(i) if $\varphi$ is bounded below, then $\left(A_{3}\right)$ is satisfied;

(ii) if $\varphi$ is bounded above, then $\left(A_{4}\right)$ is satisfied.

Proof. (i) Let $\left\{x_{n}\right\} \subset X$ be an increasing sequence. It suffices to show that $\left\{x_{n}\right\}$ is a Cauchy sequence. If otherwise, there exists an increasing subsequence $\left\{x_{n_{i}}\right\} \subset\left\{x_{n}\right\}$ and $\delta>0$ such that

$$
d\left(x_{n_{i}}, x_{n_{i+1}}\right) \geq \delta, \quad \forall i .
$$

Then by $\left(A_{9}\right)$ and $\left(A_{12}\right)$, we have

$$
\varphi\left(x_{n_{i}}\right)-\varphi\left(x_{n_{i+1}}\right) \geq \eta(\delta), \quad \forall i,
$$

which implies that $\left\{\varphi\left(x_{n_{i}}\right)\right\}$ is a decreasing sequence of reals and hence convergent since $\varphi$ is bounded below. Moreover by (19), we have

$$
\varphi\left(x_{n_{i+1}}\right) \leq \varphi\left(x_{n_{1}}\right)-i \eta(\delta), \quad \forall i .
$$

Let $i \rightarrow \infty$, and by $\eta^{-1}(\{0\})=\{0\}$ we have $\lim _{i \rightarrow \infty} \varphi\left(x_{n_{i}}\right)=$ $-\infty$, which is a contradiction. And consequently, $\left\{x_{n}\right\}$ is a Cauchy sequence.

(ii) Let $\left\{x_{n}\right\} \subset X$ be a decreasing sequence. It suffices to show that $\left\{x_{n}\right\}$ is a Cauchy sequence. If otherwise, there exists a decreasing subsequence $\left\{x_{n_{i}}\right\} \subset\left\{x_{n}\right\}$ and $\delta>0$ such that (18) is satisfied. Then by $\left(A_{9}\right)$ and $\left(A_{12}\right)$, we have

$$
\varphi\left(x_{n_{i+1}}\right)-\varphi\left(x_{n_{i}}\right) \geq \eta(\delta), \quad \forall i,
$$

which implies that $\left\{\varphi\left(x_{n_{i}}\right)\right\}$ is an increasing sequence of reals and hence convergent since $\varphi$ is bounded above. Moreover by (21), we have

$$
\varphi\left(x_{n_{i+1}}\right) \geq \varphi\left(x_{n_{1}}\right)+i \eta(\delta), \quad \forall i .
$$

Let $i \rightarrow \infty$, and by $\eta^{-1}(\{0\})=\{0\}$ we have $\lim _{i \rightarrow \infty} \varphi\left(x_{\alpha_{n_{i}}}\right)=$ $+\infty$, which is a contradiction. And consequently, $\left\{x_{n}\right\}$ is a Cauchy sequence. The proof is complete.

By Lemma 8, Remark 6 and (i) of Remark 7, we have the following two corollaries.

Corollary 9. Let $(X, d)$ be a complete metric space, let $\preceq$ be a partial order on $X$, and let $T: X \rightarrow 2^{X}$. Assume that $\left(A_{9}\right)-\left(A_{12}\right)$ are satisfied, $\varphi$ is a bounded below (resp., bounded above) functional, there exists $x_{0} \in X$ such that $T x_{0} \cap\left[x_{0},+\infty\right) \neq \varnothing$ (resp., $\left.T x_{0} \cap\left(-\infty, x_{0}\right] \neq \varnothing\right)$, and $T$ is increasing (resp., quasi-increasing) and has compact value on $\left[x_{0},+\infty\right)\left(\right.$ resp.,$\left.\left(-\infty, x_{0}\right]\right)$. Then $T$ has a maximal fixed point $x^{*} \in\left[x_{0},+\infty\right)$ (resp., a minimal fixed point $\left.x_{*} \in\left(-\infty, x_{0}\right]\right)$.

Corollary 10 (see [6, Theorem 5]). Let $(X, d)$ be a complete metric space, and let $\leq$ be a partial order on $X$. Assume that $\left(A_{9}\right),\left(A_{10}\right)$, and $\left(A_{12}\right)$ are satisfied and $\varphi$ is a bounded below functional. Then each set-valued Caristi-type mapping $T$ : $X \rightarrow 2^{X}$ has fixed point in $X$.
In analogy to the proof of [6, Lemma 1], we can prove the following lemma by (i) of Remark 7.

Lemma 11. Let $(X, d)$ be a complete metric space and let $\leq$ be a relation on $X$ introduced by (17). Assume that $\left(A_{8}\right)$ is satisfied. Then

(i) if $\varphi$ is lower semicontinous on $X$, then $\left(A_{10}\right)$ is satisfied;

(ii) if $\varphi$ is upper semicontinuous on $X$, then $\left(A_{11}\right)$ is satisfied;

(iii) if $\varphi$ is continuous, then $\left(A_{5}\right)$ is satisfied.

The following two corollaries directly follow from Corollaries 9 and 10, Lemma 11, and Remark 7.

Corollary 12. Let $(X, d)$ be a complete metric space, let $\leq$ be a relation on $X$ introduced by (17), and let $T: X \rightarrow 2^{X}$. Assume that $\left(A_{8}\right)$ is satisfied, $\varphi: X \rightarrow \mathbb{R}$ is a continuous and bounded below (resp., bounded above) functional, there exists $x_{0} \in X$ such that $T x_{0} \cap\left[x_{0},+\infty\right) \neq \varnothing\left(\right.$ resp., $\left.T x_{0} \cap\left(-\infty, x_{0}\right] \neq \varnothing\right)$, and $T$ is increasing (resp., quasi-increasing) and has compact value on $\left[x_{0},+\infty\right)\left(\right.$ resp., $\left.\left(-\infty, x_{0}\right]\right)$. Then $T$ has a maximal fixed point $x^{*} \in\left[x_{0},+\infty\right)$ (resp., a minimal fixed point $\left.x_{*} \in\left(-\infty, x_{0}\right]\right)$.

Corollary 13 (see [3, Theorem 4.2]). Let $(X, d)$ be a complete metric space and let $T: X \rightarrow 2^{X}$. Assume that $\left(A_{8}\right)$ is satisfied, $\varphi: X \rightarrow \mathbb{R}$ is a lower semicontinuous and bounded below functional, and for each $x \in X$, there exists $y \in T x$ such that $\eta(d(x, y)) \leq \varphi(x)-\varphi(y)$. Then $T$ has fixed point in $X$.

Remark 14. (i) Theorems 3 and 4 in [8] are special cases of Corollary 12 with $\eta(t)=t$.

(ii) Note that each single-valued mapping $T: X \rightarrow X$ naturally has compact value on $X$. Then (i) of both Theorems 1 and 2 in [10] immediately follows from Corollary 9, and Theorem 2 in [9] is directly derived from Corollary 12. Moreover, if $\eta(t)=t$, then Corollary 12 is reduced to Theorems 3 and 4 in [7].

Remark 15. It follows from Remark 2 in [6] that generalized Caristi's fixed point theorems obtained by Feng and Liu [3], Khamsi [4], and Li [5] are equivalent to primitive Caristi's result [1] and all the relating results in [3-5] could be obtained by Corollary 10; contrarily, Corollary 10 could not be derived from the results of [3-5]. Therefore, Theorem 4 indeed improves the results in [3-6].

\section{Conflict of Interests}

The authors declare that they there is no conflict of interests.

\section{Authors' Contribution}

The authors have contributed in obtaining the new results presented in this paper. All authors read and approved the final paper. 


\section{Acknowledgments}

The work was supported by Natural Science Foundation of China (11161022), Natural Science Foundation of Jiangxi Province (20114BAB211006, 20122BAB201015), Educational Department of Jiangxi Province (GJJ12280, GJJ13297), and Program for Excellent Youth Talents of JXUFE (201201).

\section{References}

[1] J. Caristi, "Fixed point theorems for mappings satisfying inwardness conditions," Transactions of the American Mathematical Society, vol. 215, pp. 241-251, 1976.

[2] W. A. Kirk, "Caristi's fixed point theorem and metric convexity," Colloquium Mathematicum, vol. 36, no. 1, pp. 81-86, 1976.

[3] Y. Feng and S. Liu, "Fixed point theorems for multi-valued contractive mappings and multi-valued Caristi type mappings," Journal of Mathematical Analysis and Applications, vol. 317, no. 1, pp. 103-112, 2006.

[4] M. A. Khamsi, "Remarks on Caristi's fixed point theorem," Nonlinear Analysis: Theory, Methods \& Applications, vol. 71, no. 1-2, pp. 227-231, 2009.

[5] Z. Li, "Remarks on Caristi's fixed point theorem and Kirk's problem," Nonlinear Analysis: Theory, Methods \& Applications, vol. 73, no. 12, pp. 3751-3755, 2010.

[6] Z. Li and S. Jiang, "Maximal and minimal point theorems and Caristi's fixed point theorem," Fixed Point Theory and Applications, vol. 2011, article 103, 2011.

[7] X. Zhang, "Fixed point theorems of monotone mappings and coupled fixed point theorems of mixed monotone mappings in ordered metric spaces," Acta Mathematica Sinica, vol. 44, no. 4, pp. 641-646, 2001 (Chinese).

[8] X. Zhang, "Fixed point theorems of multivalued monotone mappings in ordered metric spaces," Applied Mathematics Letters, vol. 23, no. 3, pp. 235-240, 2010.

[9] Z. Li, "Fixed point theorems in partially ordered complete metric spaces," Mathematical and Computer Modelling, vol. 54, no. 1-2, pp. 69-72, 2011.

[10] S. Jiang and Z. Li, "Largest and least fixed point theorems of increasing mappings in partially ordered metric spaces," Fixed Point Theory and Applications, vol. 2013, article 74, 2013. 


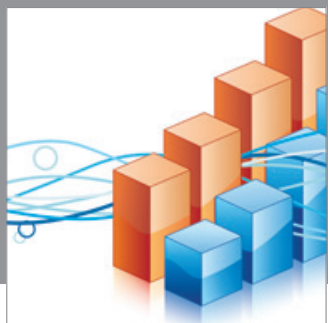

Advances in

Operations Research

mansans

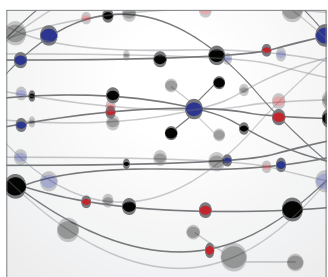

The Scientific World Journal
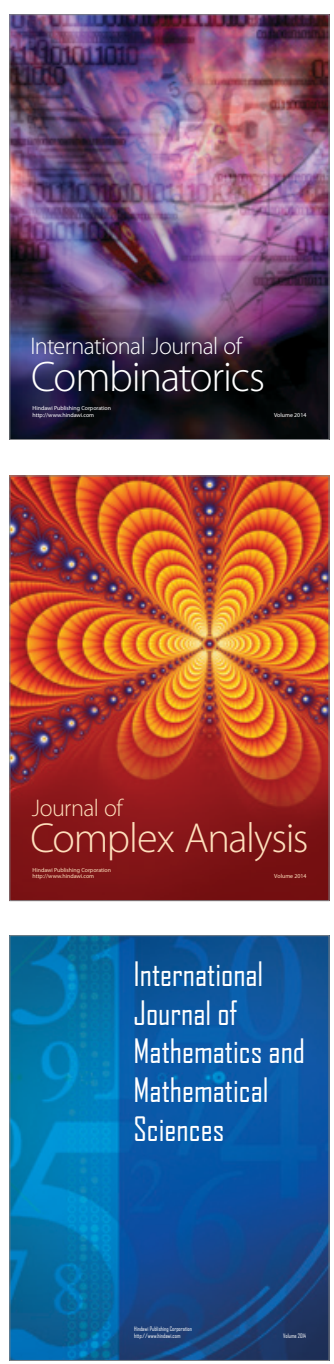
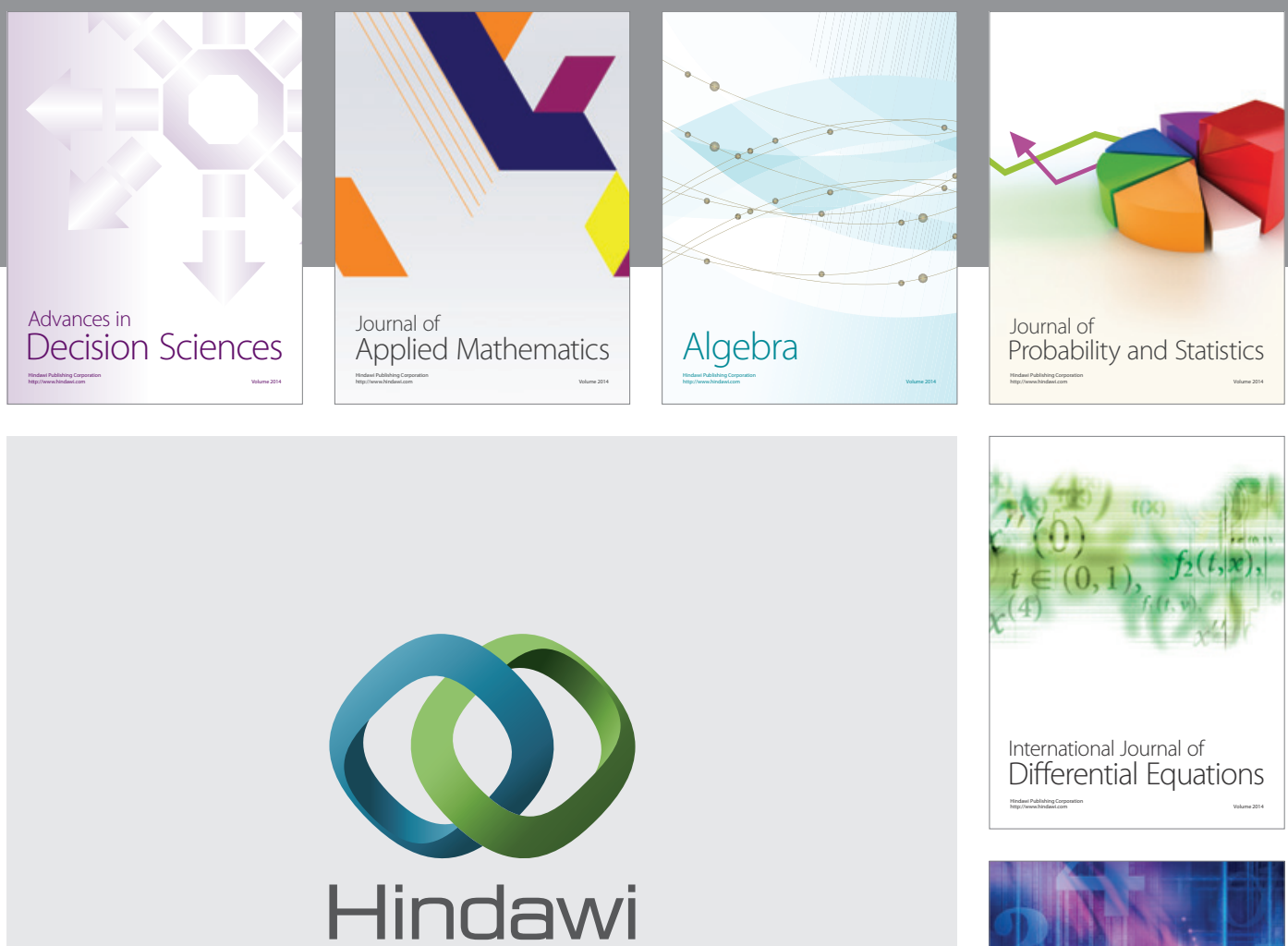

Submit your manuscripts at http://www.hindawi.com
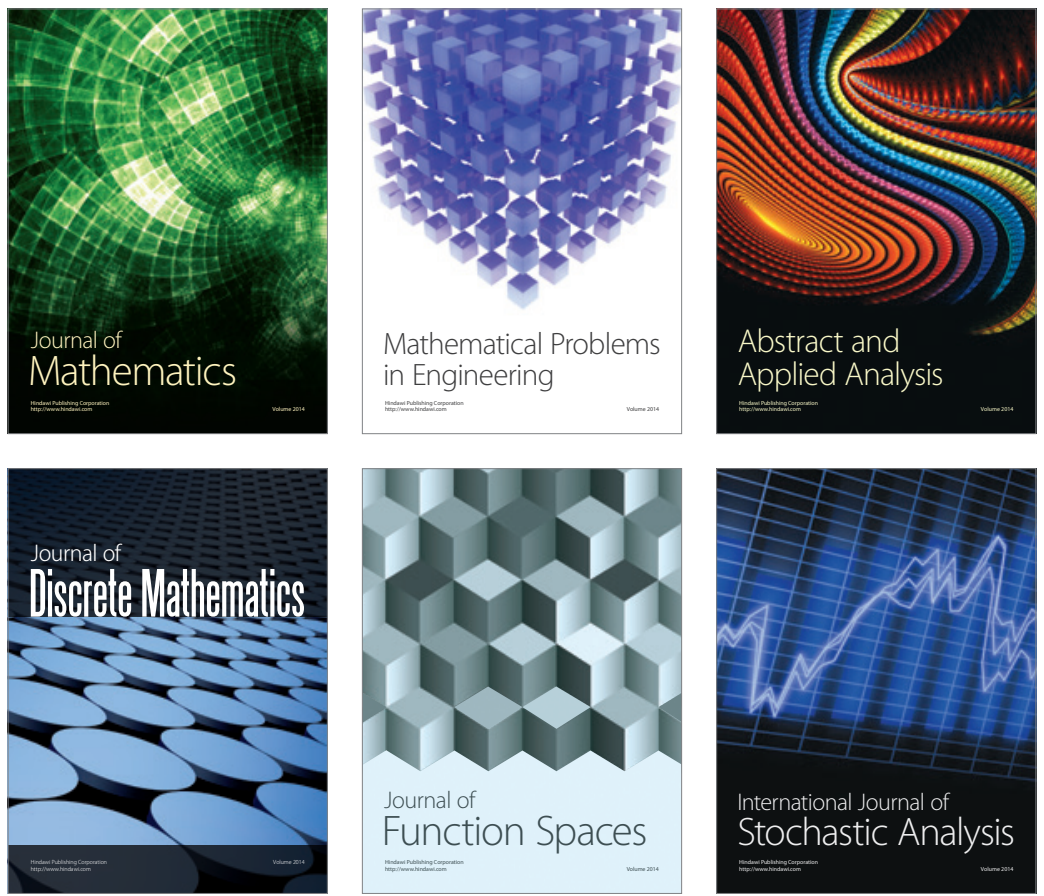

Journal of

Function Spaces

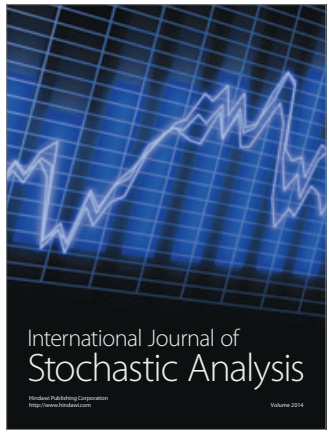

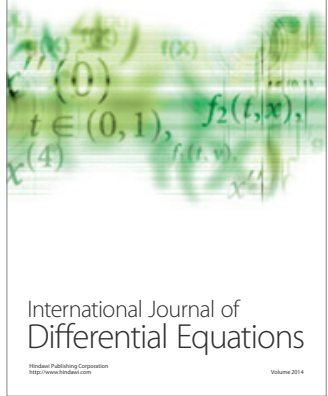
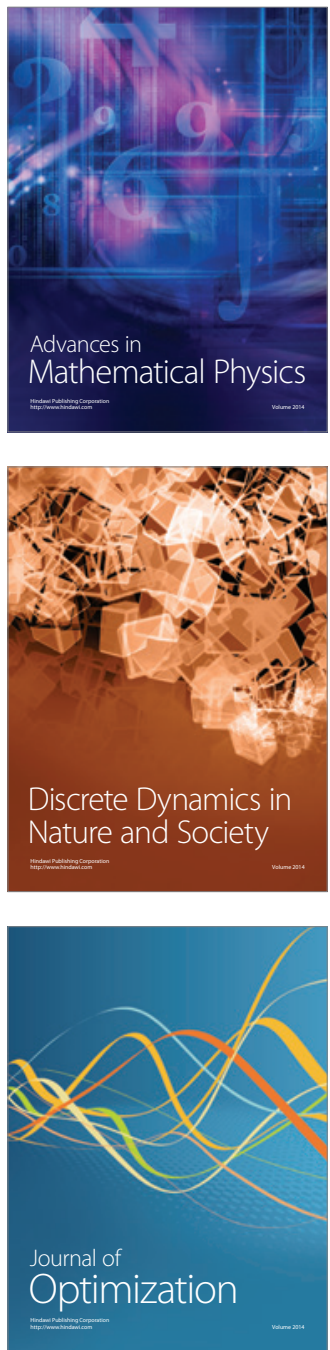